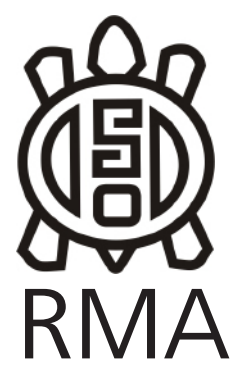

Dossier

\section{Análisis de los conjuntos ictioarqueológicos recuperados en sitios del Chaco Húmedo argentino}

Mariano Santini*

*Facultad de Ciencias Naturales y Museo, Universidad Nacional de La Plata, CONICET. E-mail: marianosantini@yahoo.com.ar

\begin{abstract}
Resumen
Este trabajo tiene como objetivo presentar y discutir la variabilidad que exhiben los conjuntos ictioarqueológicos recuperados en cinco sitios arqueológicos ("El Cachapé Potrero V", "El Cachapé Potrero IV A", "El Cachapé Potrero IV B", "Sotelo I" y "Puesto Fantin"), todos ellos localizados próximos a cursos de agua de diferente importancia en la región del Chaco Húmedo argentino (pcia. de Chaco). Cronológicamente los sitios se encuentran datados entre los 1700-900 años $C^{14}$ AP. Para el análisis de los conjuntos ictioarqueológicos, se realizó su identificación y cuantificación, tanto taxonómica como anatómica, además de evaluarse diferentes aspectos tafonómicos que pudieron haber influido tanto en el origen como en la conservación de los mismos. Se valoró la importancia de estos recursos en el marco general de la subsistencia humana para cada uno de los sitios, en particular, y para la región, en general. De los conjuntos analizados se infiere que los peces constituyeron, en todos los casos, un recurso complementario en la dieta, aunque se evidencia una mayor importancia para los sitios "El Cachapé Potrero IV A", "El Cachapé Potrero IV B" y "Puesto Fantin".
\end{abstract}

Palabras claves: Peces; Subsistencia; Holoceno tardío; Chaco Húmedo; Cuenca Paraguay-Paraná.

Analysis of the ichthyoarchaeological assemblages recovered in sites of the argentinian Humid Chaco

\begin{abstract}
The aim of this paper is to present and discuss the variability exhibited for the ichthyoarchaeological assemblages recovered in five archaeological sites ("El Cachapé Potrero V", "El Cachapé Potrero IV A", "El Cachapé Potrero IV B", "Sotelo I" and "Puesto Fantin"), all located next to different important streams in the Humid Chaco region of Argentina (Chaco province). Chronologically, the sites are dated between $1700-900{ }^{14} \mathrm{CBP}$. For the analysis of the ichthyoarchaeological assemblages, taxonomic, anatomical identification, and quantification was performed. Different taphonomic aspects that could have influenced their origin and preservation were also evaluated. The importance of these resources within the overall framework of human subsistence for each site was assessed in particular, and in general for the region. From the sets analyzed, it is inferred that fish constituted, in all cases, as a complementary resource in the diet, although there is evidence of greater importance for the sites "El Cachapé Potrero IV A", "El Cachapé Potrero IV B" and "Puesto Fantin".
\end{abstract}

Keywords: Fishes; Subsistence; Late Holocene; Humid Chaco; Paraguay-Paraná basin.

El Chaco Húmedo argentino comenzó a ser investigado sistemáticamente a partir del año 2000 y desde entonces se han realizado numerosos aportes sobre diversos aspectos de las sociedades que habitaron esta región, tales como tecnología cerámica (Lamenza et al. 2011), subsistencia (Santini 2009, 2011; Santini et al. 2010), modos de inhumación, movilidad, asentamiento y cronología (Balbarrey et al. 2010; Lamenza et al. 2007; Santini 2009), entre otros. El estado actual de las investigaciones en el Chaco Húmedo da cuenta de una importante ocupación humana en la región, identificándose hasta el momento más de 20 sitios arqueológicos, todos ellos ocupados temporalmente durante el Holoceno tardío desde por lo menos los 1700 años AP. Esta ubicación temporal está en concordancia con lo registrado por otros autores para ocupaciones a lo largo de toda la cuenca ParaguayParaná-Plata de la Argentina (Acosta 2005; Loponte 2008; Pérez Jimeno 2006; entre otros). En esta gran cuenca, los peces han sido de suma importancia en la subsistencia de los grupos humanos (Acosta y Musali 2002; Loponte 2008; Musali 2005; Pérez Jimeno 2006; entre otros). Asimismo, en todos los sitios estudiados por nosotros en este sector del Chaco, se ha recuperado hasta el momento una importante cantidad de restos de peces.

El presente trabajo tiene como objetivo presentar los resultados del análisis realizado en los conjuntos ictiofaunísticos de los sitios "El Cachapé Potrero V", "El Cachapé Potrero IV A" y "El Cachapé Potrero IV B", "Sotelo I" y "Puesto Fantin" (Figura 1), localizados 


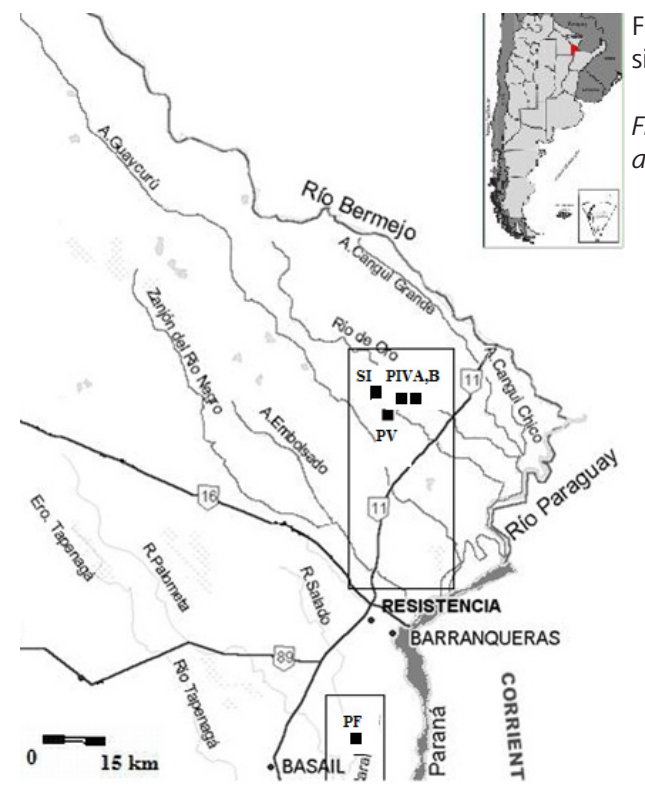

próximos a cursos de agua de diferente importancia en el área de influencia de la cuenca Paraguay-Paraná en la región del Chaco Húmedo argentino (pcia. de Chaco), con el fin de evaluar la importancia de los peces en la subsistencia de las poblaciones humanas que habitaron el Chaco Húmedo argentino. Para ello se describen en forma sintética algunos aspectos generales de los conjuntos arqueofaunísticos del área de estudio, para luego presentar los resultados obtenidos del análisis ictioarqueológico efectuado en los sitios. Asimismo, se busca contribuir a la discusión de problemas que exceden los límites regionales.

\section{Ambiente y recursos}

El área donde se localizan los sitios arqueológicos pertenece a la Provincia Fitogeográfica del Chaco, Distrito Oriental. La misma se ubica en el Sector Ribereño Paraguay-Paraná del Gran Chaco Meridional, específicamente en lo que se conoce como Subregión de Esteros, Cañadas y Selvas de Ribera y en los Bajos de Tacuarí (Morello y Adámoli 1974), que incluye toda la superficie comprendida entre los ríos autóctonos de la provincia del Chaco tributarios de los ríos ParaguayParaná, dentro del Chaco Húmedo (Burkart et al. 1999). La región oriental, denominada también Chaco Húmedo, se distingue por su exceso de humedad, resultado de una combinación de factores pluviométricos, topográficos y edáficos, donde los valores de precipitaciones superan las pérdidas por evapotranspiración. Esto permite la formación de una gran diversidad de ambientes, como bosques, palmares, pastizales, bañados, cañadas, riachos, esteros (Figura 2). Además, es una zona que se caracteriza por ser una llanura extremadamente plana. Todo ésto se traduce en una alta biodiversidad. En términos zoogeográficos, se encuentra en el Distrito Chaqueño del Dominio Subtropical de la Subregión Guayano-Brasileña (Ringuelet 1961). La gran variedad de ambientes mencionados hace que se presente en la región una notable diversidad y cantidad de fauna silvestre. Entre

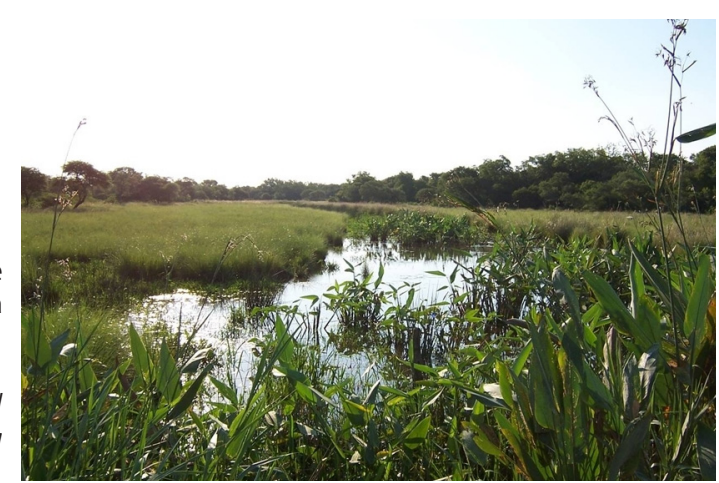

los vertebrados se encuentran más de 150 especies de peces, 40 de anfibios, 50 de reptiles, 350 de aves y 70 de mamíferos. Desde el punto de vista ictiofaunístico el área de estudio se encuentra dentro del Dominio Paranaense (Ringuelet 1975) y también podría ser englobada dentro de la región ictiográfica denominada Eje Potámico Subtropical (López et al. 2002). Los cursos de agua y espejos temporarios y permanentes albergan una variedad muy interesante y diversa de peces (Menni et al. 1992), con más de 100 especies (sin considerar el río Paraguay), predominando claramente los representantes de los Órdenes Characiformes y Siluriformes. Dentro del primero se destacan Salminus maxillosus (dorado), Prochilodus platensis (sábalo) y Hoplias malabaricus malabaricus (tararira). Dentro del segundo sobresalen los bagres de las familias Pimelodidae (bagres blancos y amarillo; P. albicans y maculatus), Doradidae (conocidos como armados, entre otros, Pterodoras granulosus), Loricadidae (viejas de agua, Hypostomus sp., Liposarcus anisitsi) y Callichthynae (vulgarmente conocidos como cascarudos, Hoplosternum littorale, Lepthoplosternum pectorales). Resulta interesante la presencia de Synbranchus marmoratus (anguilas criollas) y la existencia de una especie de pez pulmonado endémica de la región, Lepidosiren paradoxa.

\section{Metodología}

La determinación taxonómica y anatómica del material ictioarqueológico se desarrolló mediante un enfoque comparativo con muestras osteológicas actuales de especies de peces presentes en el área de estudio. En este caso se contó con la ayuda del Lic. Sergio Bogan. Con el propósito de establecer la abundancia relativa de los taxones representados y de sus partes esqueletarias se emplearon las medidas de abundancia NISP, MNI, MNE y MAU\% (Lyman 1994). Para el diagnóstico de marcas y huellas, en términos generales, se utilizaron los trabajos de Lyman (1994) y Mengoni Goñalons (1999). Mientras que para evaluar el origen de las fracturas presentes en las espinas pectorales se siguieron los criterios presentados por González (2005). Para evaluar la representación de partes esqueletales se realizaron correlaciones entre los índices de densidad ósea existentes para $P$. granulosus (Musali et al 2003) y los MAU\% de las tres especies mayormente representadas en el registro, teniendo en consideración las claras diferencias existentes en cuanto a tamaño y robustez que presentan los elementos de 


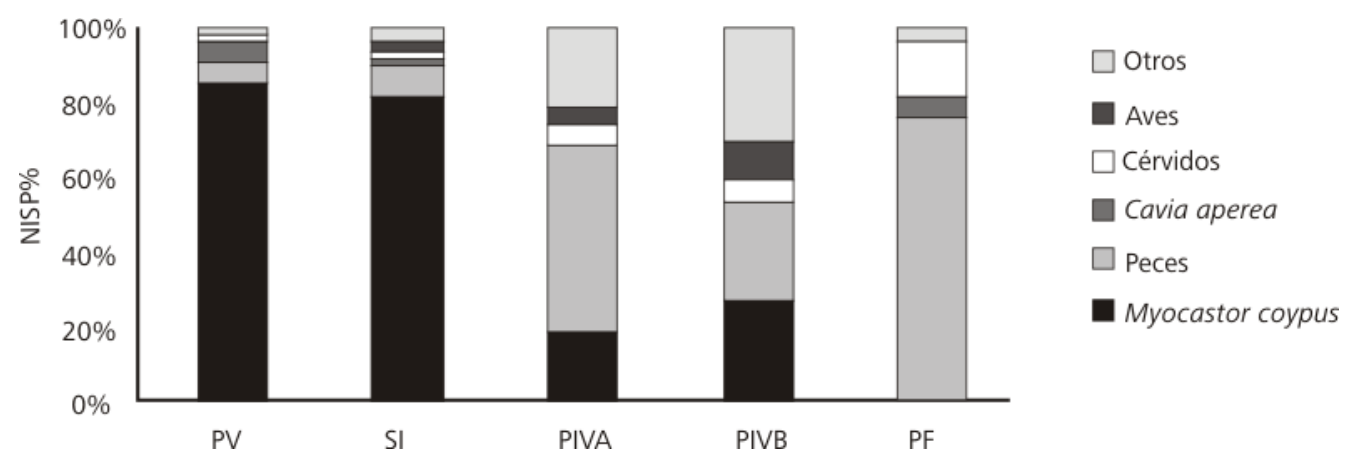

Figura 3. NISP\% de las principales presas representadas en los conjuntos arqueofaunísticos

Figure 3. NISP\% of the principal prey represented in the archaeofaunal assemblages. esta especie con las evaluadas aquí. Con el propósito de determinar la posible existencia de algún patrón de selección relacionado con el tamaño corporal de las presas obtenidas, se midió la altura de la cabeza de articulación de las espinas pectorales para las tres especies mayormente representadas en los conjuntos ( $P$. albicans, $P$. granulosus, y $L$. anisitsi) y luego se aplicó el Test "t de Student" para evaluar la existencia de diferencias estadísticamente significativas entre los sitios ( $p \leq 0,05)$. En los cinco sitios, el sedimento extraído fue cernido en seco en zarandas con malla metálica de 5 y $3 \mathrm{~mm}$. No fue posible embolsar el fondo de zaranda para posteriormente cernirlo en húmedo. EI MNI de los peces del Orden Siluriformes se obtuvo, principalmente, mediante la cuantificación de las espinas pectorales considerando la lateralidad, mientras que para el resto de los peces identificados se utilizaron diferentes elementos del cráneo. La riqueza taxonómica fue evaluada mediante la sumatoria del número de taxones ( $\sum$ NTAXA) evitando su superposición (Grayson 1991).

\section{Resultados}

El registro faunístico recuperado en los sitios “El Cachapé Potrero V" (PV) (1200 \pm 60 años AP, LP-1730), "Sotelo I" (SI) (1690 \pm 90 años AP, LP-1917), "El Cachapé Potrero IV A" (PIVA) (1260 \pm 80 años AP, LP-1780), “El Cachapé Potrero IV B" (PIVB) (1680 \pm 100 años AP, LP-1734) y "Puesto Fantin" (PF) (930 \pm 70 años AP, LP-1566), se caracteriza por la presencia de ciervo de los pantanos

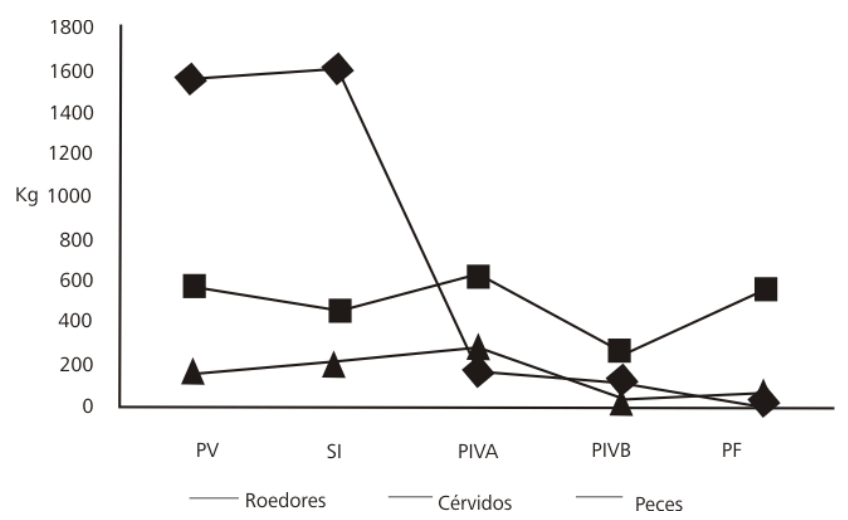

Figura 4. Biomasa estimada en kilogramos a partir de los MNI de los principales recursos explotados en los sitios

Figure 4. Estimated biomass in kilograms from the MNI of the principal resources exploited in the sites.
(Blastocerus dichotomus), venado de las pampas (Ozotoceros bezoarticus), corzuela (Mazama sp.), pequeños roedores (Myocastorydae, Caviidae), peces (Siluriformes y Characiformes) y otra serie de mamíferos y reptiles pertenecientes a las Familias Didelphidae, Canidae, Teiidae, entre otras (Figura 3). El registro arqueofaunístico de los sitios estudiados en el área muestra dos modalidades, una representada en los sitios PV y SI, donde se observa una explotación preferencial de Myocastor coypus y $B$. dichotomus y, de forma complementaria, Mazama sp., Cavia aperea, la pesca y la recolección de moluscos. Y la otra, reconocida en los sitios PIVA, PIVB y PF, donde la subsistencia está basada en la explotación de los cérvidos, complementada por una proporción más significativa de peces (Figura 4), donde la recolección de moluscos, aunque presente, no es significativa. Las especies reconocidas a través del análisis de los conjuntos óseos muestran una importante selectividad respecto de la potencial gama de presas que pudieron ser aprovechadas por las poblaciones humanas.

En primera instancia, con el propósito de determinar el probable origen de los conjuntos ictioarqueológicos y establecer en qué medida pudieron haber estado afectados por la depositación natural, es necesario realizar una serie de consideraciones tafonómicas (Colley 1990; Zohar et al. 2001; Acosta y Musali 2002; Acosta et al. 2007). Se han desarrollado diferentes investigaciones basadas en estudios comparativos entre los conjuntos ícticos formados por humanos y aquellos de origen natural (Steward y Gifford-González 1994), en las cuales se ha señalado que la depositación natural de peces tiende a ser muy baja. En los sitios estudiados, se ha observado que, en términos de NISP/MNI, los peces representan entre el 8 y el $75 \%$ de los restos faunísticos totales recuperados. Por su parte, entre las posibles causas citadas en la literatura de incorporación natural de los peces a los conjuntos óseos, se encuentra la muerte y depositación de restos por inundaciones que pudieran afectar los albardones (Acosta et al. 2004). Sin embargo, cuatro de los sitios (PV, SI, PIV A y B) se encuentran en ambientes de muy baja energía, que no fueron afectados por acción fluvial (ver Santini 2009). Asimismo, este tipo de inundaciones no es habitual, registrándose una sola en los últimos 50 años (com. pers. moradores de la zona), la cual no alcanzó los cuatro albardones en cuestión. Otra posible causa de ingreso natural es la depositación 


\begin{tabular}{|c|c|c|c|c|c|c|c|c|c|c|}
\hline \multirow{2}{*}{ Taxón } & \multicolumn{2}{|c|}{ PV } & \multicolumn{2}{|c|}{ SI } & \multicolumn{2}{|c|}{ PIVA } & \multicolumn{2}{|c|}{ PIVB } & \multicolumn{2}{|c|}{ PF } \\
\hline & NISP & MNI & NISP & MNI & NISP & MNI & NISP & MNI & NISP & MNI \\
\hline $\begin{array}{l}\text { Liposarcus anisitsi(vieja de } \\
\text { agua) }\end{array}$ & 148 & 47 & 238 & 74 & 216 & 63 & 35 & 20 & 38 & 17 \\
\hline $\begin{array}{l}\text { Pimelodus albicans(bagre } \\
\text { blanco) }\end{array}$ & 28 & 6 & 9 & 4 & 188 & 55 & 17 & 6 & 19 & 4 \\
\hline Pimelodus sp. (bagre) & 61 & 16 & 88 & 22 & 16 & 28 & 23 & 4 & 87 & 13 \\
\hline Pterodoras granulosus(armado) & - & - & 12 & 7 & 97 & 16 & 15 & 3 & 49 & 7 \\
\hline $\begin{array}{l}\text { Hoplosternum } \\
\text { litorale(cascarudo) }\end{array}$ & 13 & 3 & 45 & 21 & 59 & 12 & 5 & 4 & 22 & 8 \\
\hline Prochilodus lineatus(sábalo) & 2 & 1 & - & - & 18 & 4 & - & - & - & - \\
\hline Trachelyopterus sp.(torito) & 6 & 2 & 4 & 2 & 15 & 4 & 1 & 1 & 10 & 3 \\
\hline $\begin{array}{l}\text { Lepidosiren } \\
\text { paradoxa(lepidosirena) }\end{array}$ & 57 & 19 & 23 & 8 & 12 & 4 & 3 & 1 & 7 & 3 \\
\hline $\begin{array}{l}\text { Pimelodus maculatus(bagre } \\
\text { amarillo) }\end{array}$ & 4 & 2 & 19 & 6 & 11 & 6 & - & - & 23 & 9 \\
\hline $\begin{array}{l}\text { Synbranchus } \\
\text { marmoratus(anguila criolla) }\end{array}$ & 141 & 64 & 18 & 9 & 10 & 4 & 1 & 1 & 11 & 6 \\
\hline Serrasalmus sp. (palometa) & 6 & 2 & - & - & 10 & 6 & - & - & 4 & 1 \\
\hline Salmonus brasiliensis (dorado) & 2 & 1 & - & - & 10 & 2 & - & - & - & - \\
\hline Leporynus sp. (boga) & - & - & - & - & 9 & 2 & - & - & - & - \\
\hline Hoplias malabaricus(tararira) & 6 & 1 & - & - & 8 & 2 & 1 & 1 & 2 & 1 \\
\hline Anadoras sp.(armado) & 6 & 3 & - & - & 7 & 3 & - & - & 24 & 8 \\
\hline Pseudoplatystoma sp.(surubí) & - & - & - & - & 6 & 5 & - & - & - & - \\
\hline Leporynus obtusidens(boga) & - & - & - & - & 2 & 1 & - & - & - & - \\
\hline Otros & - & - & 4 & 2 & 17 & 4 & 2 & 1 & 10 & 1 \\
\hline Actinopterygii & 206 & - & 27 & - & 280 & - & 33 & - & 511 & - \\
\hline Total & 686 & 167 & 487 & 155 & 991 & 221 & 136 & 42 & 817 & 81 \\
\hline
\end{tabular}

Tabla 1. Abundancia taxonómica de los conjuntos ictioarqueológicos

Table 1. Taxonomic abundance of the ictioarchaeological assemblages

MNI estimado en 167, para el sitio PV; 487 y 155 en SI; 991 y 221 en PIVA; 136 y 42 en PIVB; y 817 y 81 en PF (Tabla 1). El 66\% de los restos fue determinado a nivel de especie y/o género. Se logró una alta proporción de restos determinados a nivel de especie y/o género debido, principalmente, a la presencia de gran cantidad de elementos de la cintura escapular (especialmente cleitros $\mathrm{y}$ espinas pectorales $\mathrm{y}$ dorsales, Tablas 2 y 3), elementos diagnósticos de primer orden y de fácil determinación en peces del Orden Siluriformes $y$, como se muestra más adelante, a la escasa de egagrópilas de aves (Andrews 1990; Wheeler y Jones 1989) y excrementos de carnívoros (Vuillermoz y Sapoznikow 1998), sin embargo, en los sitios en cuestión, no se han registrado marcas y modificaciones que puedan correlacionarse con estos procesos. Un mecanismo natural que pudo haber tenido un efecto agregativo menor, es la depositación por la predación de aves y posterior descarte de los cadáveres sobre el sitio, hecho observado en el sitio PF y sus inmediaciones, con restos de peces pertenecientes a Liposarcus anisitsi. Por todo lo anteriormente expuesto, se considera que existe una baja probabilidad de que los conjuntos ictioarqueológicos se encuentren sobredimensionados de manera significativa por la depositación natural de huesos. Por último, los restos presentan una buena integridad concordante con lo registrado para los restos óseos pertenecientes a mamíferos, lo que estaría indicando su rápido enterramiento.

El macrotaxón Peces es el que presenta el NISP\% más elevado en los sitios PIVA, B y PF, llegando a representar más del $50 \%$ de los restos óseos totales recuperados en este último (Figura 3). Se han identificado para los cinco sitios un mínimo de ocho especies y un máximo de 19 (Tabla 1), constituidas en su mayoría por especies de pequeño a mediano tamaño corporal. El total de especímenes recuperados (NISP) fue de 686, con un representación de las vértebras, elementos de escaso valor diagnóstico.

Las especies mayormente representadas en los cinco sitios pertenecen al Orden Siluriformes, de las cuales las que presentan mayor frecuencia de NISP y MNI son Liposarcus anisitsi (vieja de agua), individuos de la familia Pimelodidae (bagre blanco y amarillo) seguidos por Synbranchus marmoratus (anguila) y el pez pulmonado Lepidosiren paradoxa en los sitios PV y SI y Pterodoras granulosus en los restantes sitios (Tabla 1).

La representación de partes esqueletarias de las especies anteriormente mencionadas, es similar en todos los conjuntos analizados. Los restos del esqueleto axial, tales como cráneo, mandíbula y vértebras, se encuentran en muy bajas proporciones (menos del $20 \%$ de las muestras). Un dato llamativo, compartido por todos los sitios arqueológicos del norte de la provincia de Chaco, es la escasa cantidad de vértebras recuperadas ( $P V=128$, $\mathrm{SI}=10, \mathrm{PIV} A=184, \mathrm{PIV} B=29$ ), mientras que en Puesto Fantin representan más del $50 \%$ de los restos $(n=426)$. Cabe recordar, que en todos los sitios se utilizó la misma metodología de tamizado (mallas de zaranda de 3 y 5 $\mathrm{mm}$ ). En los peces Characiformes sólo se encuentran muy pocos elementos craneales, mientras que en los Siluriformes, la representación esqueletal es más 


\begin{tabular}{lccccc}
\hline \multicolumn{1}{c}{$\begin{array}{l}\text { Elemento } \\
\text { espina }\end{array}$} & PV & SI & PIVA & PIVB & PF \\
pectoral & 87 & 143 & 100 & 29 & 22 \\
espina dorsal & 43 & 9 & 33 & 4 & 4 \\
cleitro & 3 & 4 & 10 & 2 & 2 \\
coracoide & 1 & - & 1 & - & - \\
frontal & 6 & 1 & 9 & - & - \\
supraoccipital & 2 & 1 & 12 & - & - \\
placas & & & & & \\
cráneo & 2 & 5 & 30 & - & - \\
otros & 4 & - & 18 & - & - \\
\hline
\end{tabular}

\begin{tabular}{lccccc}
\hline \multicolumn{1}{c}{ Elemento } & PV & SI & PIVA & PIVB & PF \\
espina pectoral & 39 & 47 & 79 & 13 & 47 \\
espina dorsal & 19 & 24 & 20 & 3 & 25 \\
cleitro & 16 & 36 & 113 & 10 & 34 \\
supracleitro & 1 & - & 8 & - & 2 \\
coracoide & 1 & - & 3 & - & - \\
frontal & 3 & 2 & 21 & 1 & 2 \\
opercular & 1 & 2 & 8 & 3 & 7 \\
subopercular & - & - & 3 & - & - \\
supraoccipital & 6 & - & 15 & 2 & 4 \\
basioccipital & - & - & 1 & - & - \\
etmoide lateral & 1 & - & 2 & 1 & - \\
placas cráneo & 1 & - & 30 & - & - \\
dentario & - & 4 & 1 & 1 & - \\
otros & - & - & 21 & - & 7 \\
\hline
\end{tabular}

homogénea. Dadas las características etológicas de las especies presentes, es altamente probable que los peces hayan ingresado enteros al sitio. Se trata de especies que se encuentran en riachos y cuerpos de agua de tipo léntico, tales como los que se observan lindantes a los sitios arqueológicos. Son peces frecuentadores de aguas quietas, con abundante vegetación, sumergida y flotante, y presentan gran capacidad para sobrevivir en momentos de sequía (Menni et al. 1992; Menni 2004).

Con el propósito de dilucidar si esta representación de partes esqueletarias podría estar mediada por la densidad mineral ósea se realizaron correlaciones con los estudios de densidad mineral ósea realizados para $P$. granulosus (Siluriforme) y $P$. lineatus (Characiforme) (Musali et al. 2003). En todos los casos las correlaciones dieron resultados altamente significativos, sugiriendo que la supervivencia diferencial de los elementos presentes se podría deber a su densidad mineral ósea (Tabla 4). Los elementos mayormente representados son las espinas pectorales y dorsales y algunos huesos craneales, los cuales presentan las densidades más altas, mientras que los menos abundantes son las vértebras, las que presentan las densidades más bajas. Es de destacar que las vértebras son el elemento que mayor frecuencia tiene en todos los conjuntos ictioarqueológicos presentes a lo largo de la cuenca del Plata (Acosta 2005; Loponte 2008; Pérez Jimeno 2006), ya que si bien su densidad mineral es baja, su morfología las convierte en elementos con elevadas probabilidades de supervivencia (Musali et al. 2003). Así, su ausencia en cuatro de los cinco sitios estudiados puede estar mediada por otras circunstancias no relacionadas con su densidad mineral (véase discusión).

No se registraron especímenes con huellas de corte aunque si se recuperaron, en todos los sitios, instrumentos óseos utilizando como soportes espinas pectorales y dorsales de peces del Orden Siluriformes $(P V=3, S I=5$, PIV $A=2, P I V B=1, P F=3$ ) (Figura 5).

A partir de la medición de la altura de la cabeza de articulación de las espinas pectorales de $P$. albicans, P. granulosus, y L. anisitsi, los porcentajes obtenidos para los cinco sitios indican una prevalencia de entre el $47,3 \%$ y el $67,5 \%$ hacia la selección de presas de tamaño mediano, las que presentan carillas articulares entre 10 y $14 \mathrm{~mm}$, sin embargo, se visualiza una tendencia a capturar en promedio presas de mayor tamaño corporal en los sitios PIV A, B y PF (Tabla 5). Estas diferencias son estadísticamente significativas entre estos tres sitios y PV y SI, tanto para $L$. anisitsi como para $P$. granulosus, mientras que para $P$. albicans las diferencias no son significativas, aunque puede apreciarse un promedio de captura de ejemplares mayores.

En todos los conjuntos, cerca del 30\% del total de espinas pectorales de Pimelodidae y $P$. granulosus se encuentran fragmentadas en estado fresco, mientras que para $L$. anisitsi, alrededor del $37 \%$ presenta fracturas pero en estado seco. A partir de observaciones actuales en pescadores del Paraná se ha planteado la posibilidad de la fractura intencional de las espinas pectorales como medida precautoria para evitar lastimaduras en los pescadores (Acosta y Musali 2002). Esta hipótesis es válida para especies de las familias Doradidae y Pimelodidae, las cuales presentan un gran desarrollo denticular, sin

\begin{tabular}{llcc}
\hline \multicolumn{1}{c}{ Sitio } & \multicolumn{1}{c}{ Especie } & r-Spearman & \multicolumn{1}{c}{$\mathbf{P}$} \\
PV & Pimelodus sp. & 0,778 & $<0,01$ \\
& L. anisitsi & 0,852 & $<0,01$ \\
SI & Pimelodus sp. & 0,745 & $<0,01$ \\
& L. anisitsi & 0,863 & $<0,01$ \\
PIV A & Pimelodus sp. & 0,652 & $<0,05$ \\
& P. granulosus & 0,768 & $<0,01$ \\
& L. anisitsi & 0,863 & $<0,01$ \\
PIV B & Pimelodus sp. & 0,736 & $<0,01$ \\
PF & Pimelodus sp. & 0,693 & $<0,05$ \\
& P. granulosus & 0,731 & $<0,01$ \\
& L. anisitsi & 0,82 & $<0,01$ \\
\hline
\end{tabular}

Tabla 4. Correlación entre el MAU\% y los índices de densidad mineral ósea.

Table 4. Correlation between MAU\% and the bone mineral density indexes. 


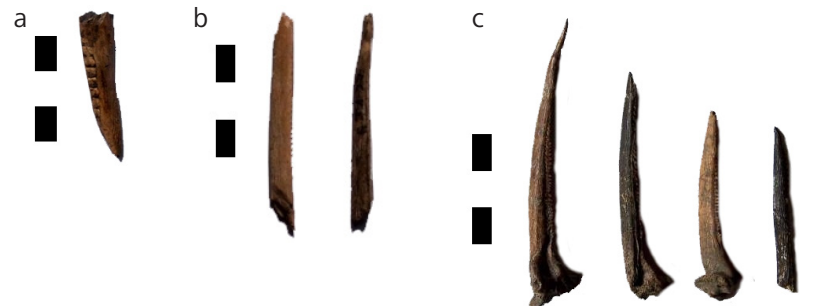

Figura 5a, b y c. Instrumentos confeccionados sobre espinas pectorales recuperados en los sitios PV, PF y SI.

Figure $5 a, b$, and $c$. Instruments made on pectoral spines recovered at sites $P V, P F$, and $S I$.

embargo, para el caso de las espinas pectorales de la Familia Loricaridae, que no presentan dentículos, la fractura podría deberse a la baja resistencia que presentan a la presión mecánica, fragmentándose por procesos tafonómicos.

Por último, menos del $2 \%$ de los restos presentan evidencias de termoalteración, aunque este hecho podría estar subdimensionado debido a la presencia de precipitación de óxido de manganeso sobre los conjuntos.

\section{Discusión y Consideraciones finales}

La información proveniente de estudios de diversidad específica y biomasa realizados en ambientes lénticos (bañados y cursos de agua menor), similares a los presentes en cercanías de los sitios estudiados (Menni et al. 1992; Menni 2004), muestran como resultado la existencia de una mayor oferta ambiental de especies pertenecientes al Orden Characiformes en relación al Orden Siluriformes. A partir de las características eco-etológicas de las especies mayormente presentes en el registro ictioarqueológico, se desprende que su lugar de captura habría ocurrido en los cursos de agua y bañados de las inmediaciones de las bases residenciales, no habiéndose explotado especies de mayor porte y biomasa (sábalo, pacú, boga, entre otras), como las que se encuentran en los ríos Guaycurú, de Oro y Paraná, ubicados a distancias menores a los 15 $\mathrm{km}$ de los sitios. En general, la representación de especies estaría indicando que su captura se habría dado en las proximidades de los sitios, ya que se trata de especies que se encuentran en riachos y cuerpos de agua de tipo léntico. Son peces frecuentadores de aguas quietas y con abundante vegetación, sumergida y flotante, y todos ellos presentan gran capacidad para sobrevivir en momentos de sequía. En los sitios PIVA, B y PF, ubicados próximos a cursos de agua de mayor importancia, se observa una explotación de especímenes de talla promedio superior.

La elevada diversidad de especies registradas en todos los sitios, su abundancia, accesibilidad, y el tamaño de las principales especies representadas, podría explicarse a través de la utilización de diferentes estrategias para la obtención de los peces. Así, los habitantes de los sitios pudieron desplegar métodos de pesca indiscriminados para maximizar su retorno por el esfuerzo invertido, tal

\begin{tabular}{lccccc}
\hline \multicolumn{1}{c}{ Taxón } & PV & SI & PIV A & PIV B & PF \\
P. granulosus & - & 9,44 & 12,98 & 13,2 & 13,3 \\
L. anisitsi & 10,5 & 11,2 & 11,7 & 12,2 & 12,1 \\
P. albicans & 10,41 & 11,07 & 11,51 & 11,92 & 12,6 \\
\hline
\end{tabular}

Tabla 5. Tamaño promedio expresado en milímetros, de las carillas articulares de espinas pectorales de las especies más representadas del Orden Siluriformes

Table 5. Average size expressed in millimeters of the pectoral spines articular facets from the greater represented species of Siluriformes.

como el uso de redes y/o trampas naturales, que hubieran permitido capturar de manera oportunística una gran diversidad de especies y tamaños corporales (Reitz y Masucci 2004), como las registradas en los conjuntos ictioarqueológicos. Por otro lado, la captura de L. anisitsi y Pimelodus sp., especies caracterizadas por habitar en aguas poco profundas y/o nadar cerca de la superficie, se pudo haber llevado a cabo a través de la recolección manual y/o arponeo en sectores con niveles de agua de poca profundidad. Las fuentes etnohistóricas para la región, mencionan el uso de numerosas técnicas para la captura de los peces, tales como anzuelos, arpones, redes y trampas, incluso la recolección manual (Dobrizhoffer 1967 TI: 424, 425,426- TII: 128).

Arqueológicamente, inferimos la existencia de redes (Balbarrey et al. 2010; Santini 2011) que pudieron utilizarse tanto para la caza como para la pesca, a partir de la presencia de decoración en la cerámica de improntas de cordelería. Además, se recuperaron en los sitios instrumentos clasificados morfológicamente como arpones y puntas, los que pudieron utilizarse para la captura de las presas, aunque su número es bajo (Santini y Plischuk 2006; Santini 2009).

Como fuera planteado con anterioridad (Santini et al. 2010), uno de los datos más llamativos, compartido por todos los sitios arqueológicos ubicados al norte de la provincia de Chaco, es la escasísima presencia de vértebras en los conjuntos recuperados. Por su parte, en Puesto Fantin, ubicado al sur de la provincia, estos elementos representan más del $50 \%$ del NISP para peces, dato similar a los registrados para sitios ubicados a lo largo del Paraná medio e inferior (Acosta 2005; Pérez Jimeno 2006; entre otros), con los que comparte, tanto en la tecnología ósea como en el material cerámico (ver Calandra et al. 2004, Liggera et al. 2011), características más afines que con aquellos sitios localizados hacia el norte (río Paraguay). Las posibles causas de la baja representación de estas unidades anatómicas podrían ser: a) un posible sesgo producido por el tamaño de las mallas utilizadas en el cernido del sedimento y la imposibilidad de guardar y analizar el fondo de zaranda; b) los métodos de preparación de los alimentos, tales como la producción de harina de pescado o el ahumado de las presas.

En cuanto al posible sesgo producido por la imposibilidad 
de guardar y estudiar el fondo de zaranda, el cual ha demostrado que eleva el número de vértebras recuperadas (véase discusión en Loponte 2008), análisis posteriores en que se extraigan columnas de muestreo permitirán determinar si hubo o no un sesgo metodológico en la recolección. Por su parte, es de destacar que en todos los sitios analizados se utilizó la misma metodología, tamizando con zarandas de 5 y $3 \mathrm{~mm}$, y sólo en uno de ellos (Puesto Fantin) el número de vértebras es elevado, por lo que la presencia diferencial podría deberse a diferentes técnicas de procesamiento y consumo de los peces.

La utilización de técnicas culinarias que permiten diferir el consumo de los alimentos, entre ellas, el ahumado, secado por oreo y harina, podrían ser una explicación de la ausencia de vértebras en los conjuntos de los sitios PV, SI, PIV A y B. Por un lado, el ahumado y secado permitirían amortiguar las variaciones ambientales que pudieran producirse, consumiendo las presas en otros campamentos o fuera de los mismos, mientras se realizan otras actividades o durante los traslados. Por otro lado, la preparación de harina de pescado podría implicar el molido y consecuente destrucción de las vértebras durante su procesamiento, técnica utilizada actualmente por grupos Tobas y Wichis del Chaco (véase Arenas 2003: 489).

Para finalizar hay que recordar que en ninguno de los conjuntos ictioarqueológicos analizados se registraron huellas de corte, hecho descripto recurrentemente en la literatura arqueológica para peces de talla pequeñamediana (Acosta 2005; Steward y Gifford-González 1994; entre otros).

Si bien los peces constituyeron la presa más numerosa en términos de NISP y MNI en tres de los cinco sitios analizados, no han cumplido el mismo papel central en la dieta como parece haber sucedido entre los grupos humanos ubicados a lo largo del Paraná medio e inferior (Acosta 2005; Loponte 2008; Musali 2005). Sin embargo, es evidente que los peces constituyeron un recurso complementario de importancia en todos los sitios, en general, y en PIV A, B y PF, en particular, donde la explotación de peces adquirió una mayor importancia relativa ante una disminución significativa en la explotación de roedores, especialmente de $M$. coypus.

La Plata, 14 de Noviembre de 2011

\section{Agradecimientos.}

Quisiera agradecer a los coordinadores de la mesa de poster del II CNZA, Lic. Álvarez y Lic. Rafuse. Asimismo agradezco los comentarios y sugerencias realizadas por los evaluadores.

\section{Bibliografía}

Acosta, A. 2005 "Zooarqueología de cazadores-recolectores del extremo nororiental de la provincia de Buenos Aires (humedal del río Paraná inferior, Región Pampeana, Argentina)". Tesis Doctoral inédita. Facultad de Ciencias Naturales y Museo. Universidad Nacional de La Plata, Argentina.

Acosta, A. y J. Musali. 2002. Ictioarqueología del sitio La Bellaca 2 (Pdo. de Tigre, Pcia. de Buenos Aires). Informe preliminar. Intersecciones en Antropología 3: 3-16.

Acosta, A; D. Loponte, S. Duran, L. Mucciolo, J. Musali, L. Pafundi y D. Pau. 2004. Albardones naturales vs. culturales: exploraciones tafonómicas sobre la depositación natural de huesos en albardones del nordeste de la provincia de Buenos Aires. Aproximaciones Arqueológicas Pampenas. Teorías, Métodos y Casos de Aplicación Contemporáneos, pp. 77-92 editado por G. Martínez, M. Gutiérrez, R. Curtoni, M. Berón y P. Madrid, Facultad de Ciencias Sociales, UNCPBA.

Acosta A., D. Loponte y J. Musali. 2007. A Taphonomic Approach to the Ichtyoarchaeological Assemblage of La Bellaca site 2, Wetland of the Lower Paraná River, Pampean Region (Argentina). En Taphonomy and Zooarchaeology in Argentina, editado por M. Gutiérrez, G. Barrientos, G. Mengoni Goñalons, L. Miotti y M. Salemme: 71-88. B.A.R. International Series 1601, Oxford.

Andrews, A. P. 1990. Owls, caves and fossils. University of Chicago Press, Chicago.

Arenas, P. 2003. Etnografía y alimentación entre los TobaÑachilamolek y Wichí-Lhuku'tas del Chaco Central (Argentina). Buenos Aires. pag. 348.

Balbarrey G., G. Lamenza, M. Santini, C. De Feo, H. A. Calandra y S. A. Salceda. 2010. Espacio Social y Territorialidad de Sociedades Prehispánicas del Chaco Argentino. Folia Histórica del Nordeste, N¹8: 137-150.

Burkart, R., N. Bárbaro, R. Sánchez y D. Gómez. 1999. Ecoregiones de la Argentina. Administración de Parques Nacionales, Programa Desarrollo Institucional Ambiental, Buenos Aires.

Calandra H. A., M. Santini, S. Salceda, G. Lamenza. 2004. Arqueología ribereña del Chaco: Presentación de un nuevo sitio arqueológico. XXIV Encuentro de Geohistoria Regional: 104-108. Resistencia, Chaco.

Colley, S. M. 1990. The análisis and interpretation of archaeological fish remains. Archaeological Method and Theory 2:207-253.

Dobrizhoffer, M. 1967 Historia de los abipones. Tomo I y II. Universidad Nacional del Noroeste, Facultad de Humanidades, Departamento de Historia, Resistencia.

González, M. I. 2005 Arqueología de Alfareros, Cazadores y Pescadores Pampeano. Sociedad Argentina de Antropología, Buenos Aires. 
Grayson, D. K. 1991. Alpine faunas from the White mountains, California: Adaptive Change in the Late Prehistoric Great Basin?. Journal of Archaeological Science 18: 483-506.

Lamenza G., M. Santini y J. Castro. 2007. Ocupación del espacio en el Sector Ribereño Paraguay-Paraná (Chaco-Argentina). $2^{\circ}$ Encuentro de Discusión Arqueológica del Nordeste Argentino. Paraná.

Lamenza, G; G. Balbarrey y H. Calandra. 2011. Estudio preliminar de los restos cerámicos hallados en el sitio arqueológico Sotelo I (Chaco, Argentina). Ed. M. R. Feuillet Terzaghi, M. B. Colasurdo, J. I. Sartori y S. Escudero. En Avances y Perspectivas en la Arqueología del Nordeste. Pp 94-105. Ed. ST Servicios Gráficos, Santo Tomé.

Liggera, I., Lamenza G, y H. Calandra. 2011. Análisis preliminar de los restos cerámicos del sitio arqueológico Puesto Fantin (Chaco, Argentina). Trabajo presentado en el XXXI Encuentro de Geohistoria Regional. UADE, Concepción de Uruguay, Entre Ríos.

López, H.L; C. C. Morgan y M. J. Montenegro. 2002. Ichthyological Ecoregions of Argentina. Probiota Serie Documentos № 1.

Loponte, D. 2008. Arqueología del humedal del Paraná inferior (bajios ribereños meridionales). Asociación Amigos del Instituto Nacional de Antropología y Pensamiento Latinoamericano, Buenos Aires.

Lyman, R. L. 1994. Vertebrate Taphonomy. Cambridge: Cambridge University Press.

Mengoni Goñalons, G. L. 1999. Cazadores de guanacos de la estepa patagónica. Sociedad Argentina de Antropología. Colección Tesis Doctorales. Buenos Aires.

Menni, R.C. 2004. Peces y ambientes en la Argentina continental. Monografías del Museo Argentino de Ciencias Naturales 5. Estudio Sigma, Buenos Aires, 316 p.

Menni, R.C., A. M. Miquelarena, H. L. Lopez, J. R. Casciotta, A. E. Almirón y C.C. Protogino. 1992. Fish fauna and environmentes of the Pilcomayo-Paraguay basins in Formosa, Argentina. Hydrobiol 245: 142-146.

Morello, J. y Adámoli, J. 1974. Las grandes unidades de vegetación y ambiente del chaco argentino. Segunda parte: vegetación y ambiente de la Provincia del Chaco. Secretaría de Estado de Agricultura y Ganadería de la Nación. INTA, Serie Fitogeográfica No 13. Buenos Aires. 130 pp

Musali, J. 2005. "Ictioarqueología del Delta de río Paraná inferior". Tesis de Licenciatura inédita en Ciencias Antropológicas (orientación Arqueológica), Facultad de Filosofía y Letras, Universidad de Buenos Aires, Argentina.

Musali, J., A. Acosta y D. Loponte. 2003. Methodological issues: an attempt to asses bone representation of Pampa's Wetlands ichthyoarchaeological record, Argentina. Memories of the ICAZ FRWG: 17-123. Guadalajara, Mexico.

Pérez Jimeno, L. 2006. "Investigaciones arqueológicas en el sector septentrional de la llanura alluvial del Paraná -margen santafesina-: La variabilidad del registro arqueológico". Tesis de Doctorado. Facultad de Ciencias Naturales y Museo. Universidad Nacional de La Plata, Argentina.

Reitz, E. J. y M. A. Masucci. 2004. Guangala. fishers and Farmers. A Case Study of Animal Use at El Azúcar, Southwestern Ecuador. University of Pittsburg Memoirs in Latin American Archaeology No14.

Ringuelet, R. A. 1961. Rasgos fundamentales de la zoogeografía de la Argentina. Physis 22 (63): 151-170.

Ringuelet, R. A. 1975. Zoogeografía y ecología de los peces de aguas continentales de la Argentina y consideraciones sobre las áreas ictiológicas de América del Sur. Ecosur, Vol 2 (3): 1-122.

Santini, M. 2009. "Prehistoria de la región meridional del Gran Chaco: aportes del análisis de restos faunísticos en la reconstrucción de las estrategias adaptativas de los grupos aborígenes durante el Holoceno tardío". Tesis de Doctorado inédita. Facultad de Ciencias Naturales y Museo. Universidad Nacional de La Plata, Argentina.

Santini, M. 2011. Aprovechamiento de Myocastor coypus en sitios del Chaco Húmedo argentino. Intersecciones en Antropología (12): 195-205.

Santini, M. y M. Plischuk. 2006. Sector Ribereño Paraguay-Paraná: análisis de los conjuntos de artefactos óseos provenientes de dos sitios arqueológicos. Actas del XXVI Encuentro de Geohistoria Regional, pp. 491-495. Resistencia, Chaco.

Santini M, S. Salceda y J. M. De Santis. 2011 Análisis del registro arqueofaunístico del sitio Sotelo I (provincia del Chaco). Ed. M. R. Feuillet Terzaghi, M. B. Colasurdo, J. I. Sartori y S. Escudero. En Avances y Perspectivas en la Arqueología del Nordeste. Pp 3-25. Ed. ST Servicios Gráficos, Santo Tomé.

Stewart, M. y D. Gifford-González. 1994. An ethnoarchaeological contribution to identifying hominid fish processing sites. Journal of Archaeological Science 21: 237-248.

Vuillermoz, P. y A. Sapoznikow. 1998. Hábitos alimenticios y selección de presas de los carnívoros medianos en la Reserva de Vida Silvestre "Campos del Tuyu". Boletín Técnico 44, Fundación Vida Silvestre. Buenos Aires.

Wheeller, A. y A. K. Jones. 1989. Fishes. Cambridge University Press, New York.

Zohar, I., T. Dayan, E. Galili y E. Spanier. 2001. Fish Processing During the Early Holocene: A Taphonomic Case Study from Coastal Israel. Journal of Archaeological Science 28:1041-105. 